\title{
The GM(1,1) Model Optimized by Using Translation Transformation Method and Its Application of Rural Residents' Consumption in China
}

\author{
Chong LIU \\ Department of Mathematics and Computing Science, Anqing Normal University, Anqing 246133, \\ China \\ E-mail: liuchong@aqtc.edu.cn \\ Cui YANG \\ Department of Mathematics and Computing Science, Anqing Normal University, Anqing 246133, \\ China \\ E-mail: yangcui@aqtc.edu.cn
}

\begin{abstract}
Conventional GM(1,1) model shows some limitations which affect directly to the model applicability as well as prediction accuracy. In order to improve the disadvantages existing in the original grey model, the authors analyze the translation transformation approach to optimize the raw sequence for grey modeling. This paper mainly carries on the proof of the existence of translation transformation from the perspective of smooth ratio and gives the method to solve the best step size of translation transformation. Then, the authors applied the grey models optimized by translation transformation to analyze the problem of the consumption of rural residents in China. As a result, the authors find GM(1,1) model optimized by translation transformation has characteristics of higher accuracy and simple calculation process and an important practical application in residents consumption.
\end{abstract}

Keywords grey theory; translation transformation; GM (1,1) model; consumption

\section{Introduction}

The grey model GM(1,1) has been developed and successfully applied to many fields in modern life such as social sciences, agriculture, traffic, transportation, marketing, business, engineering sciences etc. and achieved promising results. But as same as other forecasting models, the grey model $\operatorname{GM}(1,1)$ has some limitations. It is only applied to single exponential growth sequence, and the prediction results can achieve good accuracy. On the other hand, we can also know from the basic form of the grey prediction model that the GM(1,1) model is constructed according to the linear changing relationship between the raw data sequence and its 1-AGO sequence, but the variation usually appears in many systems is not linear between them, so many questions need to improve the $\operatorname{GM}(1,1)$ model for a higher prediction model accuracy. At present, there are some improvements for the $\operatorname{GM}(1,1)$ model as follows:

- The improvements of estimate parameters of the $\operatorname{GM}(1,1) \operatorname{model}^{[1,2]}$.

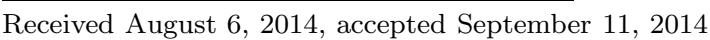


- The improvements of the background series ${ }^{[3-5]}$.

- The improvements of the initial condition of the $\operatorname{GM}(1,1)$ model ${ }^{[6,7]}$.

- The improvements of model accuracy from the form of the $\operatorname{GM}(1,1)$ model ${ }^{[8-10]}$.

Besides as above, there are a lot of improvements from the form of the $\operatorname{GM}(1,1)$ model, such as the GGM model ${ }^{[11]}$ is a simple improvement model, Zhang et al. ${ }^{[12]}$ applied derivatives to construct the grey model, Zhao et al. ${ }^{[13]}$ used a grey model optimized by differential evolution algorithm to forecast the per capita annual net income of rural households in China and $\mathrm{Wu}$ et al. ${ }^{[14]}$ presented the improvement model by using of the fractional order accumulation. Considering applicability in the case of limited data situations and requirement of little computational effort, grey prediction with rolling mechanism ${ }^{[15]}$ approach is proposed. When the data sequence changes are not smooth, the $\operatorname{SAGM}(1,1)$ model ${ }^{[16]}$ can replace the $\operatorname{GM}(1,1)$ model for higher accuracy. All these improvement methods can improve the model accuracy from a certain degree, but there is no research which fully investigates all the limitations of GM(1,1) model to design a new accurate grey prediction model with high adaptability to all application purposes. So it is necessary to find a simple approach for high model accuracy. We found the translation transformation is a very simple method, and it can be better to improve model accuracy. At present, the research on improving the grey model by using of the sequence transformation method ${ }^{[17]}$ is few, thus the studies on translation transformation for improving grey model accuracy is important significance.

The problem of residents' consumption is an important part of the study of economics, and now there are a lot of studies on the residents' consumption by using of regression analysis method ${ }^{[18-21]}$. Zhang et al. ${ }^{[22]}$ employs the Euler equation approach to examine the effect of monetary policy, conducted through the interest rate channel, on household consumption. Haddad $^{[23]}$ proposed a prediction model for recurrent consumption behaviors based on inhomogeneous Poisson processes aiming at predicting. Because the problem of residents' consumption exist information incompleteness and has a certain characteristic of exponential growth, the grey modeling method has good applicability. Comparing with other models, the grey model has a simple calculation process and high model accuracy. However, the study on residents' consumption by using of grey models is very few. In the paper, we analyzed the residents' consumption by applying grey models which optimized by the best translation transformation method.

\section{The Basic Concept of Grey Modeling}

\section{$2.1 \operatorname{GM}(1,1)$ model}

The steps of $\operatorname{GM}(1,1)$ model are shown as follows.

Step 1 Original time sequence with $n$ samples is defined as

$$
X^{(0)}=\left(x^{(0)}(1), x^{(0)}(2), \cdots, x^{(0)}(n)\right)
$$

Step 2 Construct monotonic increasing sequence $X^{(1)}$ by a one-time accumulated generating operation (I-AGO), expressed as

$$
X^{(1)}=\left(x^{(1)}(1), x^{(1)}(2), \cdots, x^{(1)}(n)\right)
$$


where

$$
x^{(1)}(k)=\sum_{i=1}^{k} x^{(0)}(i), \quad k=1,2, \cdots, n
$$

Step 3 Form the GM(1,1) model by establishing a first order grey differential equation:

$$
x^{(0)}(k)+a z^{(1)}(k)=b
$$

where $z^{(1)}(k)=\frac{1}{2}\left(x^{(1)}(k)+x^{(1)}(k-1)\right)$. In Equation $(3), k(k=2,3, \cdots, n)$ is a time point. $a$ is called the development coefficient and $b$ is called grey action coefficient. Using least mean square estimation technique coefficients, $[a, b]^{\mathrm{T}}$ can be estimated as

$$
[a, b]^{\mathrm{T}}=\left(B^{\mathrm{T}} B\right)^{-1} B^{\mathrm{T}} Y
$$

where

$$
Y=\left[\begin{array}{c}
x^{(0)}(2) \\
x^{(0)}(3) \\
\vdots \\
x^{(0)}(n)
\end{array}\right], \quad B=\left[\begin{array}{cc}
-z^{(1)}(2) & 1 \\
-z^{(1)}(3) & 1 \\
\vdots & \vdots \\
z^{(1)}(n) & 1
\end{array}\right]
$$

Step 4 According to the estimated coefficients $a$ and $b$, the grey prediction equation can be obtained by solving differential equation in Equation (3).

$$
\tilde{x}^{(1)}(k+1)=\left(x^{(1)}(1)-\frac{b}{a}\right) \mathrm{e}^{-a k}+\frac{b}{a}
$$

where $\tilde{x}^{(1)}(k)$ denotes the prediction of $x$ at $k$ time point.

Step 5 To obtain the forecasting values of $\tilde{x}^{(0)}(k), k=2,3, \cdots, n$, the inverse accumulated generating operation (I-AGO) is used to establish the following Grey model:

$$
\left\{\begin{array}{l}
\tilde{x}^{(1)}(k+1)=\left(x^{(1)}(1)-\frac{b}{a}\right) \mathrm{e}^{-a k}+\frac{b}{a} \\
\tilde{x}^{(0)}(k+1)=\tilde{x}^{(1)}(k+1)-\tilde{x}^{(1)}(k)
\end{array}\right.
$$

We can also simplify Equation (7) as

$$
\tilde{x}^{(0)}(k+1)=\left(1-\mathrm{e}^{a}\right)\left(x^{(1)}(1)-\frac{b}{a}\right) \mathrm{e}^{-a k}
$$

The advantages of grey prediction model $\operatorname{GM}(1,1)$ is easy to calculate and suitable for many problems of approximate exponential change. $\operatorname{GGM}(1,1)$ model which has more simple form is more suitable for the approximate exponential sequence and has a simple calculation process, so we compared the improved model $\operatorname{GGM}(1,1)$ with $\operatorname{GM}(1,1)$ model applied the translation transformation method in this paper.

\section{$2.2 \operatorname{GGM}(1,1) \operatorname{Model}^{[11]}$}

$\mathrm{e}^{-a}$ in Equation (8) was replaced with $\varpi$ as follows:

$$
\tilde{x}^{(0)}(k+1)=\left(1-\varpi^{-1}\right)\left(x^{(1)}(1)-\frac{b}{a}\right) \varpi^{k}
$$


We put Equation (9) into $f(\varpi)=\sum_{k=2}^{n}\left[x^{(0)}(k)-\tilde{x}^{(0)}(k)\right]^{2}$ making the function $f(\varpi)$ reach the minimum, then the variable $\varpi$ was obtained. Hence, the GGM $(1,1)$ model could be expressed as follows:

$$
\left\{\begin{array}{l}
f(\varpi)=\min \sum_{k=2}^{n}\left[x^{(0)}(k)-\tilde{x}^{(0)}(k)\right]^{2} \\
\tilde{x}^{(0)}(k+1)=\left(1-\varpi^{-1}\right)\left(x^{(1)}(1)-\frac{b}{a}\right) \varpi^{k}
\end{array}\right.
$$

where, the problem of solving the function $f(\varpi)$ belongs to the unconstrained nonlinear continuously differentiable mathematical programming, which could be solved by the GA method. The GGM $(1,1)$ model has a higher accuracy comparing with the GM $(1,1)$ model in theory. However, the GGM $(1,1)$ model has some limitations as well as the GM $(1,1)$ model is not suitable for a non-smooth data sequence.

\subsection{Testing for the Grey Model}

If the grey model achieves a certain predictive accuracy, not only the development coefficient $a$ takes a certain value, but also the error of the grey model must pass the error test. The calculation error can be defined as follows.

- The average absolute error

$$
\bar{\varepsilon}=\frac{1}{n} \sum_{i=1}^{n}\left|\tilde{x}^{(0)}(i)-x^{(0)}(i)\right|
$$

- The average relative error

$$
\bar{\varepsilon}_{r}=\frac{1}{n} \sum_{i=1}^{n}\left|\frac{\tilde{x}^{(0)}(i)-x^{(0)}(i)}{x^{(0)}(i)}\right|
$$

Generally, if the grey model passes the test that shows in Table 1, it can be applied to forecast. Otherwise, we must improve the grey model for a higher accuracy.

Table 1 The precision reference of the grey model

\begin{tabular}{cccc}
\hline Accuracy class & $\bar{\varepsilon}_{r}$ & $|a|$ & Practicability of the grey model \\
\hline Class-I & $<2 \%$ & $<0.3$ & Medium or long term \\
Class-II & $<10 \%$ & $<0.5$ & Short-term \\
Class-III & $<20 \%$ & $<0.8$ & Short-term used carefully \\
Class-IV & $\geq 20 \%$ & $\geq 0.8$ & Not applicable \\
\hline
\end{tabular}

\section{Translation Transformation}

\subsection{The Existence of Translation Transformation}

Definition 1 Assume that a non-negative increasing sequence $X=(x(1), x(2), \cdots, x(n))$ by using of translation transformation is expressed as

$$
\bar{X}=(x(1)-\tau, x(2)-\tau, \cdots, x(n)-\tau)
$$


where $\tau$ is translation step and $\tau>0$.

Definition 2 The smooth ratio of a non-negative increasing sequence $X=(x(1), x(2)$, $\cdots, x(n))$ is defined as

$$
\rho(k)=\frac{x(k)}{\sum_{i=1}^{k-1} x(i)}, \quad k=2,3, \cdots, n
$$

Obviously, the smooth ratio $\rho(k)$ is smaller, the raw sequence $X$ is more smoothly.

Property 1 For any non-negative increasing sequence $X=(x(1), x(2), \cdots, x(n))$, existing translation transformation $\tau$ can make smooth ratio $\rho(k)$ smaller.

Proof Assumed that translation transformation $\tau$ exists and there is the inequality

$$
\rho_{\tau}(k)<\rho(k)
$$

i.e.

From the inequality as above, we obtained

$$
\frac{x(k)-\tau}{\sum_{i=1}^{k-1}(x(i)-\tau)}<\frac{x(k)}{\sum_{i=1}^{k-1} x(i)}
$$

$$
\begin{aligned}
& \frac{x(k)-\tau}{\sum_{i=1}^{k-1} x(i)-(k-1) \tau}<\frac{x(k)}{\sum_{i=1}^{k-1} x(i)} \\
\Rightarrow & \frac{\left[(k-1) x(k)-\sum_{i=1}^{k-1} x(i)\right] \tau}{\sum_{i=1}^{k-1} x(i)\left[\sum_{i=1}^{k-1}(x(i)-(k-1) \tau)\right]}<0 \\
\Rightarrow & \frac{\tau}{\sum_{i=1}^{k-1}(x(i)-(k-1) \tau)}<0
\end{aligned}
$$

Then,we obtained $\tau<0$ or $\tau>\frac{\sum_{i=1}^{k-1}(x(i)}{k-1}$.

Thus, Equation (13) was satisfied, i.e. we could find the translation step $\tau$ in the condition assumed as above. The proof has been completed.

\subsection{The Best Translation Step}

If the grey models achieve higher prediction accuracy, the translation step selected must be appropriate. The steps of solving the translation step are defined as follows.

Step 1 Let $\bar{x}^{(0)}(k)=x^{(0)}(k)-\tau$, then

$$
\bar{x}^{(1)}(k)=\sum_{i=1}^{k} \bar{x}^{(0)}(i)
$$

Step 2 To build linear model as follows:

$$
\bar{x}^{(0)}(k)=\alpha \bar{x}^{(1)}(k)+\beta
$$

Hence

$$
x^{(0)}(k)-\tau=\alpha x^{(1)}(k)-\alpha k \tau+\beta
$$

Step 3 Let $c_{1}=\alpha \tau, c_{2}=\beta+\tau$, Equation (14) can be expressed as

$$
x^{(0)}(k)=\alpha x^{(1)}(k)-c_{1} k+c_{2}
$$

Step 4 According to the least squares principle, the parameters $\left[\alpha, c_{1}, c_{2}\right]^{\mathrm{T}}$ is defined as Equation (16).

$$
\left[\alpha, c_{1}, c_{2}\right]^{\mathrm{T}}=\left(B^{\mathrm{T}} B\right)^{-1} B^{\mathrm{T}} Y
$$


where

$$
Y=\left[\begin{array}{c}
x^{(0)}(1) \\
x^{(0)}(2) \\
\vdots \\
x^{(0)}(n)
\end{array}\right], \quad B=\left[\begin{array}{ccc}
-z^{(1)}(2) & -1 & 1 \\
-z^{(1)}(3) & -2 & 1 \\
\vdots & \vdots \\
z^{(1)}(n) & -n & 1
\end{array}\right]
$$

Then, we obtain the translation step $\tau=c_{1} / \alpha$.

\section{The Consumption of Urban Residents in China}

The data of the consumption of rural residents in China from 1995 to 2012 as Table 2 was obtained from National Bureau of Statistics, by which we could construct grey models to forecast the consumption of urban residents in China from 2013 to 2016.

Table 2 The consumption of urban residents in China (2001-2012)

\begin{tabular}{cccccc}
\hline Year & Consumption (yuan) & Year & Consumption (yuan) & Year & Consumption (yuan) \\
\hline 1995 & 1310.4 & 2001 & 1741.1 & 2007 & 3223.9 \\
1996 & 1572.1 & 2002 & 1834.3 & 2008 & 3660.7 \\
1997 & 1617.2 & 2003 & 1943.3 & 2009 & 3993.5 \\
1998 & 1590.3 & 2004 & 2184.7 & 2010 & 4381.8 \\
1999 & 1577.4 & 2005 & 2555.4 & 2011 & 5221.1 \\
2000 & 1670.1 & 2006 & 2829.0 & 2012 & 5908.0 \\
\hline
\end{tabular}

Firstly, the best translation step $\tau=1149.9$ was obtained according to Equation (16).

Secondly, according to Equation (4), the parameters $[a, b]^{\mathrm{T}}$ were $[-0.1026,850.0454]^{\mathrm{T}}$ from Table 2 and $[-0.1791,205.7365]^{\mathrm{T}}$ from the raw data sequence translated by $\tau$.

For the purpose of convenience of the analysis, the model $\operatorname{GM}(1,1)$ applied the translation transformation are defined as TT-GM $(1,1)$. When the parameters $[a, b]^{\mathrm{T}}$ were put into Equation (8) and Equation (10), we could construct three grey models: $\operatorname{GM}(1,1)$ model, $\operatorname{GGM}(1,1)$ model and TT-GM(1,1) model, by which the 1995-2012 data could be estimated and the errors were given as the following Table 3.

According to the data from Table 3, the actual data compared with the predictive values obtained by using of three grey models: $\operatorname{GM}(1,1), \operatorname{TT}-\operatorname{GM}(1,1)$ and $\operatorname{GGM}(1,1)$ as Figure 1.

It can be seen from Table 3 that the average prediction model errors are much smaller by using of translation transformation, and we also find from Figure 1 that the improvement grey model TT-GM $(1,1)$ is higher prediction accuracy comparing with other models. 
Table 3 The forecasting results of the three grey models

\begin{tabular}{|c|c|c|c|c|c|c|c|}
\hline Time & Actual value & $\operatorname{GM}(1,1)$ & $\varepsilon$ & TT-GM $(1,1)$ & $\varepsilon$ & $\operatorname{GGM}(1,1)$ & $\varepsilon$ \\
\hline 1995 & 1310.4 & 1310.4 & 0 & 1310.4 & 0 & 1310.4 & 0 \\
\hline 1996 & 1572.1 & 1036.7 & -535.42 & 1406.7 & -165.44 & 1017.5 & -554.58 \\
\hline 1997 & 1617.2 & 1148.6 & -468.56 & 1457 & -160.19 & 1125.4 & -491.82 \\
\hline 1998 & 1590.3 & 1272.7 & -317.62 & 1517.2 & -73.062 & 1244.7 & -345.63 \\
\hline 1999 & 1577.4 & 1410.1 & -167.27 & 1589.3 & 11.871 & 1376.6 & -200.8 \\
\hline 2000 & 1670.1 & 1562.4 & -107.68 & 1675.4 & 5.3288 & 1522.5 & -147.58 \\
\hline 2001 & 1741.1 & 1731.2 & -9.9491 & 1778.5 & 37.381 & 1683.9 & -57.189 \\
\hline 2002 & 1834.3 & 1918.1 & 83.808 & 1901.7 & 67.44 & 1862.4 & 28.106 \\
\hline 2003 & 1943.3 & 2125.3 & 181.96 & 2049.2 & 105.87 & 2059.8 & 116.52 \\
\hline 2004 & 2184.7 & 2354.8 & 170.07 & 2225.5 & 40.806 & 2278.2 & 93.462 \\
\hline 2005 & 2555.4 & 2609.1 & 53.679 & 2436.4 & -118.98 & 2519.6 & -35.753 \\
\hline 2006 & 2829 & 2890.8 & 61.849 & 2688.7 & -140.31 & 2786.7 & -42.27 \\
\hline 2007 & 3223.9 & 3203 & -20.852 & 2990.4 & -233.47 & 3082.1 & -141.78 \\
\hline 2008 & 3660.7 & 3549 & -111.74 & 3351.3 & -309.36 & 3408.8 & -251.87 \\
\hline 2009 & 3993.5 & 3932.2 & -61.263 & 3783 & -210.49 & 3770.2 & -223.34 \\
\hline 2010 & 4381.8 & 4356.9 & -24.898 & 4299.3 & -82.474 & 4169.8 & -212 \\
\hline 2011 & 5221.1 & 4827.4 & -393.67 & 4916.9 & -304.21 & 4611.8 & -609.3 \\
\hline 2012 & 5908 & 5348.8 & -559.23 & 5655.5 & -252.46 & 5100.7 & -807.35 \\
\hline $\bar{\varepsilon}$ & & & 184.9733 & & 88.8413 & & 242.1852 \\
\hline $\bar{\varepsilon}_{r}$ & & & $8.30 \%$ & & $3.54 \%$ & & $9.67 \%$ \\
\hline
\end{tabular}

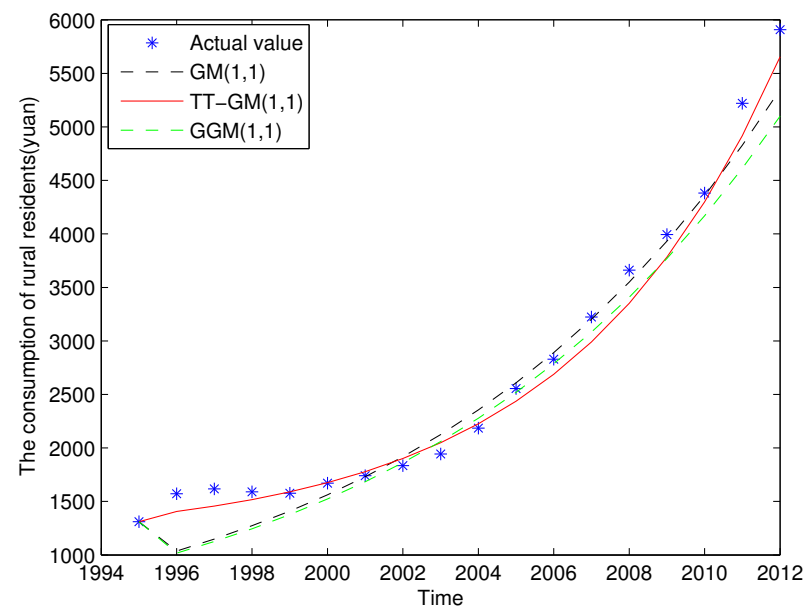

Figure 1 The actual data compared with the predictive values obtained by using of three grey models: $\operatorname{GM}(1,1), \operatorname{TT}-\operatorname{GM}(1,1)$ and $\operatorname{GGM}(1,1)$ 
Finally, the development coefficient $a$ in the TT-GM $(1,1)$ model was -0.1791 , and the average relative error $\bar{\varepsilon}_{r}$ which was $3.54 \%$ was lest among three models. According to the error test Table 1, we know that the TT-GM $(1,1)$ model was highest accuracy among three models and almost achieved Class-I, so we could put the parameter $[a, b]^{\mathrm{T}}=[0.1791,205.7365]^{\mathrm{T}}$ into Equation (8), then the consumption of rural residents in China from 2013 to 2016 which was forecasted by applying the TT-GM(1,1) model was [6539, 7595.8, 8859.7, 10372].

The actual consumption of rural residents in China 2013 is 6625.5 (yuan) from National Bureau of Statistics comparing with the predict value 6539, we know that the $\operatorname{GM}(1,1)$ model optimized by using of translation transformation can provide a very good predictive effect for the consumption of rural residents in theory. But there are many factors associated with the consumption of urban residents is unlikely to be sustained, so the TT-GM $(1,1)$ model has a high accuracy when it is only applied to short-term prediction in fact.

\section{Conclusions}

In this paper, we improved grey modeling for a class growth sequence by the best translation transformation, and applied the improved grey model $\operatorname{GM}(1,1)$ to predict the consumption of rural residents in China. We can conclude that the best translation transformation is a simple method, and easy to implement, which can improve grey model accuracy very effectively. Meanwhile, we find the best translation method is particularly suitable for improving $\operatorname{GM}(1,1)$ model to forecast similar exponential increasing sequences such as: Economic development, population and the price growth, etc.

\section{References}

[1] Deng J L. The basis of grey theory. Wuhan: Press of Huazhong University of Science and Technology, 2002, 300-309.

[2] He W Z, Song G X. Estimation of grey model parameter based on genetical algorithm. Journal of Systems Engineering, 2005, 20(4): 432-436.

[3] Tan G J. The structure method and application of background value in grey system GM(1,1) model(I). Systems Engineering — Theory \& Practice, 2000, 20(4): 98-103.

[4] Zhou P, Wei Y. The Optimization of background value in grey model GM $(1,1)$. Journal of Grey System, 2006, 9(2): 139-142.

[5] Wang Z X, Dang Y S, Liu S F, et al. The optimization of background value in $\operatorname{GM}(1,1)$ model. Journal of Grey System, 2007, 10(2): 69-74.

[6] Dang Y G, Liu S F, Liu B. The GM models that $x^{(1)}(n)$ be taken as initial value. Chinese Journal of Management Science, 2005(1): 132-134.

[7] Zhang H, Hu S G. Analysis of boundary condition for $\operatorname{GM}(1,1)$ model. Journal of Huazhong University of Science and Technology, 2001, 29(4): 110-111.

[8] Mu Y. An unbiased $\operatorname{GM}(1,1)$ model with optimum grey derivative's whitening values. Mathematics in Practice and Theory, 2003, 33(3): 13-16.

[9] Ji P R, Zhang J, Zou H B, et al. A modified unbiased GM(1,1)model. Grey Systems: Theory and Application, 2011, 1(3): 192-201.

[10] Xie N M, Liu S F. Discrete GM(1,1) and mechanism of grey forecasting model. Systems Engineering Theory \& Practice, 2005, 25(1): 93-98.

[11] Zhou W, He J M. Generalized GM $(1,1)$ model and its application in forecasting of fuel production. Applied Mathematical Modelling, 2013, 37(9): 6234-6243.

[12] Zhang Y, Wei Y, Zhou P. The improved approach of grey derivative in $\operatorname{GM}(1,1)$ model. The Journal of Grey System, 2006, 18(4): 375-380. 
[13] Zhao Z, Wang J Z, Zhao J, et al. Using a grey model optimized by differential evolution algorithm to forecast the per capita annual net income of rural households in China. Omega, 2012, 40(5): 525-532.

[14] Wu L F, Liu S F, Yao L G, et al. Grey system model with the fractional order accumulation. Communications in Nonlinear Science and Numerical Simulation, 2013, 18(7): 1775-1785.

[15] Akay D, Atak M. Grey prediction with rolling mechanism for electricity demand forecasting of Turkey. Energy, 2007, 32(9): 1670-1675.

[16] Truong D Q, Ahn K K. An accurate signal estimator using a novel smart adaptive greymodel SAGM(1,1). Expert Systems with Applications, 2012, 39(9): 7611-7620.

[17] Li C. The further generalization for grey forecasting model. Systems Engineering — Theory \& Practice, 1993, 13(1): 64-66.

[18] Wu L Y. Investigation and analysis on sports consumption of urban residents of Henan Province. Physics Procedia, 2012, 33: 2020-2026.

[19] Dong X X, Hu B C. Regional difference in food consumption away from home of urban residents: A panel data. Agriculture and Agricultural Science Procedia, 2010, 1: 271-277.

[20] Zhu Q, Peng X Z, Wu K Y. Calculation and decomposition of indirect carbon emissions from residential consumption in China based on the input-output model. Energy Policy, 2012, 48: 618-626.

[21] Zhao X L, Li N, Ma C B. Residential energy consumption in urban China: A decomposition analysis. Energy Policy, 2012, 41: 644-653.

[22] Zhang Y, Wan G H. Household consumption and monetary policy in China. China Economic Review, 2002, 13(1): $27-52$.

[23] Haddad M R, Baazaoui H, Ziou D, et al. A predictive model for recurrent consumption behavior: An application on phone calls. Knowledge-Based Systems, 2014, 64: 32-43. 